\title{
Role of Depth of Invasion in Evaluation and Management of Early-stage Oral Cavity Squamous Cell Carcinoma
}

\author{
${ }^{1}$ Bahbak Shariat-Madar, ${ }^{2}$ Jeffrey C Liu
}

\begin{abstract}
Aim: To evaluate the existing body of literature and impact of depth of invasion (DOI) in early-stage oral cavity squamous cell carcinoma (OCSCC) and its role in predicting occult cervical lymph node metastases.
\end{abstract}

Background: The prognosis for early-stage T1 to T2 disease OCSCC is relatively poor compared with other mucosal subsites within the head and neck. Primary tumor DOI can help prognosticate high-risk tumors for additional treatment.

Review results: There are unequivocal management implications in the literature demonstrating a role for elective neck dissection in early-stage OCSCC based on DOI. Following appropriate patient selection, there may be a role for sentinel lymph node biopsy in regional lymph node staging in earlystage OCSCC.

Conclusion: There are a multitude of studies demonstrating novel strategies to appropriately treat early-stage OCSCC, which are increasingly becoming standard of care. These strategies are altering the overall and disease-free survival of early-stage OCSCC. Despite advances, locoregional recurrence remains a challenge in this disease.

Clinical significance: Herein, the authors highlight a number of advances in the management of early-stage OCSCC as described in the literature, which are having an impact on disease-free and overall survival.

Keywords: Carcinoma, Cavity, Cell, Cervical, Depth, Invasion, Lymphatic, Metastases, Oral, Squamous.

How to cite this article: Shariat-Madar B, Liu JC. Role of Depth of Invasion in Evaluation and Management of Early-stage Oral Cavity Squamous Cell Carcinoma. Int J Head Neck Surg 2017;8(2):84-88.

Source of support: Nil

Conflict of interest: None

${ }^{1}$ Resident, ${ }^{2}$ Associate Professor

${ }^{1}$ Department of Otolaryngology, Head and Neck Surgery, Lewis Katz School of Medicine at Temple University, Philadelphia Pennsylvania, USA

2Department of Otolaryngology, Head and Neck Surgery, Lewis Katz School of Medicine at Temple University; Department of Surgical Oncology, Fox Chase Cancer Center, Philadelphia Pennsylvania, USA

Corresponding Author: Jeffrey C Liu, Associate Professor Department of Otolaryngology, Head and Neck Surgery, Lewis Katz School of Medicine at Temple University; Department of Surgical Oncology, Fox Chase Cancer Center, Philadelphia Pennsylvania, USA, Phone: +2157073665, e-mail: jeffrey.liu@ temple.edu

\section{INTRODUCTION}

Oral cavity squamous cell carcinoma (OCSCC) is a relatively common malignancy, with oral cavity and pharynx cancers combined impacting roughly 11.1 per 100,000 people per year, accounting for 48,330 new cases in the United States in 2016. ${ }^{1}$ Overwhelmingly, the causative agents involved in OCSCC are tobacco, including both smoking and smokeless tobacco products. Alcohol, poor oral hygiene, and the use of betel quid especially in parts of Asia are also major contributors to OCSCC. ${ }^{2,3}$ While there have been recent improvements in survival rates for OCSCC of all stages, the most dramatic improvements have occurred for those with early-stage disease. ${ }^{4}$ While the survival of early-stage OCSCC has become more favorable, early-stage OCSCC still features worse prognostic outcome than some other early-stage mucosal subsites in the head and neck, even after adjusting for comorbidities. ${ }^{5,6}$

Much of the improvement in patient survival can be attributed to efforts in improving locoregional control in early-stage disease. ${ }^{2}$ Despite steady improvements in survival of OCSCC over the past several decades, a subset of patients with clinically negative neck disease fail locoregionally, even in the face of a negative margin resection and a clinically negative neck. ${ }^{7}$ The incidence of occult nodal metastasis has been suggested to be as high as 24 and 35\% for T1 and T2 lesions respectively, in some series. ${ }^{8} \mathrm{~A}$ large series by Shah et al ${ }^{9}$ found that out of 192 elective radical neck dissections in clinically N0 necks, occult nodal metastases were noted in $34 \%$ of patients. Thus, there continues to be a need for defining clinical and pathologic factors that are predictive of occult nodal metastases.

In this article, we will briefly review the history of early-stage OCSCC management, as well as the emergence of elective neck dissection (END) for early-stage OCSCC. We will present historical efforts to establishing tumor depth of invasion (DOI) to help guide patients at risk for harboring lymph node metastasis. Finally, we review the increased interest in incorporating sentinel lymph node biopsy (SLNBx) into the treatment algorithm to minimize overtreatment and risk-stratify OCSCC.

\section{ORAL CAVITY CANCER AND METASTASIS - HISTORICAL}

Historically, management of the lymph nodes in early-stage OCSCC has included watchful waiting, postoperative 
radiation therapy, or END. ${ }^{10}$ Between the 1940s and 1970s, only modest improvement in survival outcomes was described for OCSCC in long-term retrospective studies, despite relatively aggressive surgical intervention. ${ }^{11}$ Indeed, in the 1950s and 1960s en bloc resections with neck dissection, incorporating intervening lymphatics, were commonly employed. Even early-stage disease was frequently managed with partial glossectomy and radical neck dissection into the 1970s. ${ }^{11-13}$ Although localized OCSCC lesions treated with surgery alone had nodal relapse rates as high as 35\%, tumor size alone did not predict the development of nodal failure. ${ }^{11}$ Despite a lack of clinical markers to predict subclinical neck metastases in early-stage disease, Whitehurst and Droulias ${ }^{11}$ advocated for prophylactic neck dissection or radiation therapy in patients with lesions greater than $2 \mathrm{~cm}$ in size without clinical neck disease.

In the mid-1980s, the risk for subsequent nodal relapse and failure in OCSCC was lower when patients received END over observation, with a subsequent shift toward identifying factors predictive for occult metastasis and treating this occult disease. ${ }^{14}$ Spiro et $\mathrm{al}^{14}$ had found that using T2N0 stage alone to select patients for END was inadequate. In their series, $28 \%$ of patients that underwent END were found to have pathologically positive lymph nodes, whereas $27 \%$ of patients that underwent observation subsequently developed nodal disease. By stratifying N0 patients by tumor thickness, thicker tumors predicted worse survival on multivariate analysis superseding even tumor stage. By the mid-1990s, efforts focused on identifying DOI criteria to justify END, with small series showing on multivariate analysis that a $4 \mathrm{~mm}$ DOI may be predictive in oral tongue cancers. ${ }^{15}$ Multiple studies subsequently showed varying DOI levels as appropriate predictors for risk of lymph node metastasis.

\section{ORAL CAVITY CANCER AND METASTASIS - CONTEMPORARY}

In 2009, Huang et al ${ }^{16}$ performed meta-analysis from 2009 investigating the predictive value of DOI for cervical lymph node involvement. The study pooled 1,136 patients across 16 separate studies. Odds ratios were calculated to quantify the positive and negative predictive value of tumor thickness. The study was structured to minimize the subset of patients that were falsely predicted to have negative lymph nodes in order to establish an optimal DOI cut-off. Among the studies assessed, cut-offs for tumor thickness ranged from 3 to $6 \mathrm{~mm}$ and were separated into 1-mm intervals. With increasing tumor thickness, an increase in the number of patients falsely predicted to have negative nodal disease went on to develop a positive lymph node.
However, a marked jump in the percentage of patients with falsely predicted negative nodal disease was identified between 3 and $4 \mathrm{~mm}$, with a sudden jump in that interval from 4.5 to $16.6 \%$ of patients with positive lymph node disease respectively. The authors thus recommended a tumor cut-off of $4 \mathrm{~mm}$ DOI before advocating for END.

The study had some limitations. As a meta-analysis, the study was limited by only retrospective data. Also, although subsites other than oral tongue were included in the study, there was inadequate power to evaluate the role of tumor thickness for other oral cavity subsites. It is also important to note that both DOI and tumor thickness were extracted from studies, but may have been subject to variable definitions by study. ${ }^{16}$

While the predictive quality for subclinical lymph node metastasis was well characterized by Huang et al, ${ }^{16}$ the impact of occult metastasis on outcome was examined by Ganly et al. ${ }^{10}$ Using an OCSCC database of patients treated at Memorial Sloan Kettering Cancer Center between 1985 and 2005, they employed a multivariate model to assess disease-specific and overall survival. In this study, they were able to identify important prognostic predictors of outcome across 216 patients with T1T2N0 oral tongue cancers with a median follow-up of 80 months.

This study demonstrated that occult neck metastases were one of the strongest prognostic factors in predicting worse outcome, with occult neck metastases resulting in a fivefold increase in mortality $v s$ those without occult neck disease. The authors were able to demonstrate that DOI, with a depth of $2 \mathrm{~mm}$ being used as the cut-off, predicted recurrence in the neck, with a depth greater than $2 \mathrm{~mm}$ resulting in an increase in neck recurrence by nearly fourfold. Locoregional failure, particularly nodal relapse, was found to correlate most strongly with poor outcome, and careful acknowledgement of the role of tumor DOI in predicting nodal failure was demonstrated in this study. ${ }^{10}$

The Ganly study findings were critical in illustrating that occult regional metastases carried significantly worse prognostic consequences for patients with early-stage OCSCC. In tandem with the Huang study, these two studies justified the use of END in early-stage OCSCC based on predefined DOI or tumor thickness parameters.

It is important to note, however, that both studies again focused on the oral tongue, with the Ganly study focusing solely on cancer of the oral tongue. These data may not extrapolate to other oral cavity subsites. Moreover, neither study addresses the role of either observation or adjuvant radiation therapy for these tumors.

In $2015, \mathrm{D}^{\prime} \mathrm{Cruz}$ et $\mathrm{al}^{17}$ prospectively evaluated the role of END for early oral cavity cancers. The study was 
a single institution prospective randomized controlled trial comparing elective $v s$ therapeutic neck dissection in early-stage T1/T2 OCSCC. Their study evaluated 500 patients enrolled with a median follow-up of 39 months, and found that END at 3 years demonstrated an improved rate of overall survival of $80 \%$ compared with $67.5 \%$ for therapeutic neck dissection. Moreover, there was also an improvement in disease-free survival between END and therapeutic neck dissection, at 69.5 vs $45.9 \%$ ( $\mathrm{p}<0.001)$, strongly arguing in favor of END in early-stage OCSCC.

The D'Cruz study investigated a number of different stratification factors with pre-specified subgroups, including oral cavity subsite, T1 vs T2 staging, sex, ultrasound usage, perineural invasion or lymphovascular embolization, histologic grading, radiation treatment, resection margin, and tumor depth with a cut-off of $3 \mathrm{~mm}$. END demonstrated survival benefits even after adjustment for these covariates. ${ }^{17}$

Post hoc analysis suggested a lack of benefit for END in patients with a DOI below $3 \mathrm{~mm}$, although the test of the interaction was not found to be significant. A logistic regression model for covariates found that only DOI was associated with nodal positivity, with $5.6 \%$ nodal positivity identified at $3 \mathrm{~mm}$, and $16.9 \%$ nodal positivity identified at $4 \mathrm{~mm}$. This prospective study supported a $3 \mathrm{~mm}$ cut-off for END.

Taken together, the use of DOI to identify patients at risk for nodal metastasis in oral tongue T1T2N0 OCSCC is supported by both retrospective and prospective data, with a recommendation for END in these higher risk patients. Depth of invasion has been an important clinicopathologic marker utilized in other malignancies with high risk for early nodal spread, such as cutaneous melanoma, and its emergence in the evaluation and possible future staging of oral cavity cancer will be important as the treatment standard for oral cavity cancer continues to evolve. ${ }^{10,16,17}$ The use of DOI as a criterion to select for patients in need of END has resulted in improvements in survival outcomes for patients with early-stage OCSCC. ${ }^{16,17}$

\section{THE ROLE OF SENTINEL LYMPH NODE BIOPSY}

While the literature makes it clear that DOI is an important predictor of occult metastases, there remains significant debate over the exact DOI that warrants END. Even with conservative usage of the $4 \mathrm{~mm}$ depth, the majority of END will be pathologically negative and early-stage OCSCC remains overtreated..$^{18}$ In light of this, SLNBx has emerged as a risk-stratifying procedure for early-stage OCSCC. Sentinel lymph node biopsies have historically been used in cutaneous melanoma and breast cancer; however, studies have been conducted that demonstrate a clear role for SLNBx in early-stage OCSCC SLNBx has been shown to provide high negative predictive values and sparing many patients with early-stage disease from undergoing END. ${ }^{18}$

Briefly, SLNBx involves injection of a radioactive colloid tracer adjacent to the primary tumor or scar margin. A gamma camera detects the injected radiotracer within lymphatic capillaries until it passes through or is retained within regional lymph nodes. Single-photon emission computed tomography/computed tomography can also be utilized to improve identification and visualization of sentinel lymph nodes, particularly when the SLN is close to the injection site, common in floor of mouth lesions. Following preoperative localization, the patient undergoes surgery with gamma-probe guided dissection. ${ }^{19}$ There are technical elements to this surgery, with evidence of a learning curve associated with obtaining reliable and adequate results. ${ }^{20}$

Data to support the role for SLNBx in the oral cavity were evaluated in a multicenter prospective study. ${ }^{18}$ In this study, the negative predictive value of SLNBx for T1/ T2 OCSCC in 161 patients was evaluated. Patients with T1T2N0 OCSCC were recruited over a 3-year period. Patients underwent $99 \mathrm{mTc}$-sulfur colloid injection into the lesion, with nuclear imaging, narrow-exposure SLNBx, and then completion of neck dissection. All patients enrolled, including those with negative SLNBxs, underwent completion selection neck dissection. In total, there were 106 negative SLNBxs, with 100 of those confirmed to be negative pathologically with specimens from completion neck dissection. ${ }^{18}$

The negative predictive value of this technique was $94 \%$, with T1 lesions having a predictive value of $100 \%$. The overall negative predictive value also improved to $96 \%$ with further pathological sectioning and immunohistochemistry. The advent of SLNBx in the management algorithm could spare a proportion of patients with earlystage disease but significant DOI from undergoing END. ${ }^{18}$

The technique has limitations. Adoption of the modality for oral cavity lesions is still relatively low, with a known learning curve to conducting the study and performing the procedure appropriately. One study looking at 22 centers demonstrated an overall sensitivity of $90 \%$ for SLNBx in mucosal head and neck SCCs; however, at low-volume centers, the sensitivity of the procedure was $57 \%{ }^{20}$ Thus, while SLNBx may represent an important modality in the future to sort patients into different treatment regimens, its adoption remains limited and is likely best performed at high-volume centers.

\section{DISCUSSION}

Early-stage OCSCC continues to demonstrate improvements in survival outcomes, with DOI-based criteria and the employment of END to improve locoregional control. 
While the literature supports DOI to guide selection of cases for END, debate over the appropriate DOI cut-off remains controversial. Retrospective meta-analysis data suggest $4 \mathrm{~mm}$; however, one recent prospective data favor $3 \mathrm{~mm}$.

While histopathological depth measurements can only be obtained after primary excision, in the future preoperative or intraoperative ultrasound, or other imaging technologies, may be used to assist in evaluation of the primary tumor prior to excision. ${ }^{21}$ It is also important to note that few studies have been conducted evaluating other oral cavity subsite DOI guidelines. Retrospective studies have demonstrated that $\mathrm{T} 1$ and $\mathrm{T} 2$ lesions of the mandibular gingiva have occult nodal metastases rates of 11.1 and $24.5 \%$ respectively. ${ }^{22}$ A study of early-stage gingivobuccal lesions demonstrated that a tumor thickness of $4 \mathrm{~mm}$ strongly correlated with the risk for occult nodal disease and nodal relapse. ${ }^{23}$ Palatal and maxillary alveolar lesions may also warrant END in select cases of early-stage disease. ${ }^{24}$ Future quality studies will be needed to more adequately address the role of DOI in other subsite oral cavity cancers.

Despite the clear survival benefit to the use of END in this subset of patients, it is important to note that the majority of END will be pathologically negative, and a significant subset of patients will be subjected to overtreatment of their early-stage disease. ${ }^{18}$ In light of this, SLNBx provides high negative predictive values and may spare many patients from undergoing END. ${ }^{18,20}$

Additionally, apart from its role in predicting occult nodal metastases, tumor thickness may be an independent predictor of survival. Most studies assessing DOI or tumor thickness focus primarily on locoregional failure and nodal relapse as the primary endpoint. The presence of nodal metastases upstages patients and changes their prognosis. However, tumor thickness itself appears to have independent prognostic implications. Patel et $\mathrm{al}^{25}$ demonstrated on multivariate analysis that tumor thickness of $5 \mathrm{~mm}$ or greater was an independent predictor of disease-specific survival. Pinto et $\mathrm{al}^{26}$ similarly described an independent disease-free survival advantage over 12 months for tumor thickness greater than $10 \mathrm{~mm}$. Moving forward, DOI may likely be incorporated into the next edition of AJCC staging system as a predictor of overall disease outcome.

\section{CONCLUSION}

Depth of invasion in the evaluation and management paradigm for early-stage oral cavity cancer is undoubtedly critical. While a $3 \mathrm{~mm}$ cut-off for DOI is supported by prospective data, historical data support a cut-off of $4 \mathrm{~mm}$. Further study is needed to evaluate the impact of
DOI on other oral cavity subsites. Future studies may support SLNBx as a diagnostic step during the workup of early-stage OCSCC.

\section{CLINICAL SIGNIFICANCE}

The prognostic value of DOI and the utility of END in a subset of patients based on tumor DOI have become well established. The role of DOI in prognostication and management is becoming increasingly supported for specific cut-off determinations. As the treatment paradigm continues to evolve, there may be an increasing role for SLNBx to better select patients for END.

\section{ACKNOWLEDGMENT}

Authors would like to thank the International Journal of Head and Neck Surgery for the invitation to author this descriptive review, and express our deep appreciation to Dr Jatin Shah for his extensive contributions to head and neck oncology in this issue devoted to his accomplishments.

\section{REFERENCES}

1. National Cancer Institute. T. National Cancer Institute Surveillance, Epidemiol End Results Program. Bethesda (MD): National Cancer Institute; 2017. p. 15. Available from: http://seer.cancer.gov/statfacts/html/oralcav.html (http:/ / seer.cancer.gov/statfacts/html/oralcav.html)

2. Liao C-T, Chang JT-C, Wang H-M, Ng SH, Hsueh C, Lee LY, Lin $\mathrm{CH}$, Chen $\mathrm{IH}$, Huang SF, Cheng AJ, et al. Analysis of risk factors of predictive local tumor control in oral cavity cancer. Ann Surg Oncol 2008 Mar;15(3):915-922.

3. Gillison ML. Current topics in the epidemiology of oral cavity and oropharyngeal cancers. Head Neck 2007 Aug;29(8): 779-792.

4. Schwam ZG, Judson BL. Improved prognosis for patients with oral cavity squamous cell carcinoma: analysis of the National Cancer Database 1998-2006. Oral Oncol 2016 Jan;52:45-51.

5. Habbous S, Harland LT, La Delfa A, Fadhel E, Xu W, Liu FF, Goldstein D, Waldron J, Huang SH, O'Sullivan B, et al. Comorbidity and prognosis in head and neck cancers: differences by subsite, stage, and human papillomavirus status. Head Neck 2014 Jun;36(6):802-810.

6. Sparano A, Weinstein G, Chalian A, Yodul M, Weber R. Multivariate predictors of occult neck metastasis in early oral tongue cancer. Otolaryngol Head Neck Surg 2004 Oct;131(4): 472-476.

7. Bergeron M, Gauthier P, Audet N. Decreasing loco-regional recurrence for oral cavity cancer with total Mohs margins technique. J Otolaryngol Head Neck Surg 2016 Dec;45(1):63.

8. Keski-Säntti H, Atula T, Törnwall J, Koivunen P, Mäkitie A. Elective neck treatment versus observation in patients with T1/T2 N0 squamous cell carcinoma of oral tongue. Oral Oncol 2006;42(1):96-101.

9. Shah JP, Candela FC, Poddar AK. The patterns of cervical lymph node metastases from squamous carcinoma of the oral cavity. Cancer 1990 Jul;66(1):109-113. 
10. Ganly I, Patel S, Shah J. Early stage squamous cell cancer of the oral tongue-clinicopathologic features affecting outcome. Cancer 2012 Jan;118(1):101-111.

11. Whitehurst JO, Droulias CA. Surgical treatment of squamous cell carcinoma of the oral tongue: factors influencing survival. Arch Otolaryngol 1977 Apr;103(4):212-215. Available from: http://www.ncbi.nlm.nih.gov/pubmed/849198.

12. Tulenko J, Priore RL, Hoffmeister FS. Cancer of the tongue. Comments on surgical treatment. Am J Surg 1966 Oct;112(4):562-568. Available from: http:/ / www.scopus.com/ inward / record.url?eid=2-s2.0-0013961842\&partnerID $=40 \& \mathrm{~m}$ d5=681b6f0fd19365f1098267bf68d9a7aa.

13. Johnson JT, Leipzig B, Cummings CW. Management of T1 carcinoma of the anterior aspect of the tongue. Arch Otolaryngol 1980 May;106(5):249-251.

14. Spiro RH, Huvos AG, Wong GY, Spiro JD, Gnecco CA, Strong EW. Predictive value of tumor thickness in squamous carcinoma confined to the tongue and floor of the mouth. Am J Surg 1986 Oct;152(4):345-350.

15. Asakage T, Yokose T, Mukai K, Tsugane S, Tsubono Y, Asai M, Ebihara S. Tumor thickness predicts cervical metastasis in patients with Stage I/II carcinoma of the tongue. Cancer 1998 Apr;82(8):1443-1448.

16. Huang SH, Hwang D, Lockwood G, Goldstein DP, O'Sullivan B. Predictive value of tumor thickness for cervical lymph-node involvement in squamous cell carcinoma of the oral cavity: a meta-analysis of reported studies. Cancer 2009 Apr;115(7): 1489-1497.

17. D'CruzAK, VaishR,KapreN,DandekarM,GuptaS,Hawaldar R, Agarwal JP, Pantvaidya G, Chaukar D, Deshmukh A, et al. Elective versus therapeutic neck dissection in node-negative oral cancer. N Engl J Med 2015 Aug;373:1-9.

18. Civantos FJ, Zitsch RP, Schuller DE, Agrawal A, Smith RB, Nason R, Petruzelli G, Gourin CG, Wong RJ, Ferris RL, et al. Sentinel lymph node biopsy accurately stages the regional lymph nodes for T1-T2 oral squamous cell carcinomas: results of a prospective multi-institutional trial. J Clin Oncol 2010 Mar;28(8):1395-1400.

19. Alkureishi LWT, Burak Z, Alvarez JA, Ballinger J, Bilde A, Britten AJ, Calabrese L, Chiesa C, Chiti A, de Bree R, et al. Joint practice guidelines for radionuclide lymphoscintigraphy for sentinel node localization in oral/oropharyngeal squamous cell carcinoma. Eur J Nucl Med Mol Imaging 2009 Nov;36(11):1915-1936

20. Ross GL, Shoaib T, Soutar DS, MacDonald DG, Camilleri IG, Bessent RG, Gray HW. The First International Conference on Sentinel Node Biopsy in Mucosal Head and Neck Cancer and adoption of a multicenter trial protocol. Ann Surg Oncol 2002 May;9(4):406-410.

21. Mark Taylor S, Drover C, MacEachern R, Bullock M, Hart R, Psooy B, Trites J. Is preoperative ultrasonography accurate in measuring tumor thickness and predicting the incidence of cervical metastasis in oral cancer? Oral Oncol 2010 Jan;46(1):38-41.

22. Niu LX, Feng ZE, Wang DC, Zhang JY, Sun ZP, Guo CB. Prognostic factors in mandibular gingival squamous cell carcinoma: A 10-year retrospective study. Int J Oral Maxillofac Surg 2016 Oct;46(2):137-143.

23. Mishra RC, Parida G, Mishra TK, Mohanty S. Tumour thickness and relationship to locoregional failure in cancer of the buccal mucosa. Eur J Surg Oncol 1999 Apr;25(2):186-189.

24. Morris LGT, Patel SG, Shah JP GI. High rates of regional failure in squamous cell carcinoma of the hard palate and maxillary alveolus. J Sci Spec Head Neck 2011 Jun;33(6):824-830.

25. Patel RS, Clark JR, Dirven R, Wyten R, Gao K, O'Brien CJ. Prognostic factors in the surgical treatment of patients with oral carcinoma. ANZ J Surg 2009 Jan-Feb;79(1-2):19-22.

26. Pinto FR, DeMatos LL, Palermo FC, KulcsarMA, Cavalheiro BG, de Mello ES, Alves VA, Cernea CR, Brandão LG. Tumor thickness as an independent risk factor of early recurrence in oral cavity squamous cell carcinoma. Eur Arch Otorhinolaryngol 2014 Jun;271(6):1747-1754. 